\section{Tribune libre} Freies Forum

\section{Une adresse à connaître: www.orpha-net.ch}

\author{
D. Hohl, CHUV, Lausanne
}

\section{Qu'est-ce qu'Orphanet?}

Orphanet est un serveur d'information sur les maladies rares et les médicaments orphelins, pour tous publics. Son but est de contribuer à améliorer le diagnostic, la prise en charge et le traitement des maladies rares.

Orphanet est constitué d'une encyclopédie en ligne écrite par des experts européens et d'un répertoire des services à destination des malades et des professionnels. Ce répertoire inclut des informations sur les consultations spécialisées, les laboratoires de diagnostic, les projets de recherche en cours et les associations de malades.

\section{Le Comité Scientifique Suisse}

Prof. Claude Bachmann, maladies métaboliques

Dr Armand Bottani, génétique médicale/SSGM

Prof. Jean-Michel Dayer, allergie et immunologie

Dr Michel Duchosal, hématologie

Prof. Cem Gabay, rhumatologie

Dr N. Guertler, otolaryngologie

Dr Charles A. Haenggeli, neurologie pédiatrique

Prof. Daniel Hohl, dermatologie/vénéréologie

Dr Romain Lazor, pneumologie

Dr Christoph Meier, endocrinologie/diabète

Dr Dagmar Keller, cardiologie

Dr Hans Jung, neurologie

\section{Qu'est-ce que vous pouvez trouver sur le serveur d'Orphanet?}

Dans sa version actuelle, Orphanet contient des informations sur 1150 maladies rares. Chaque maladie est décrite par un texte écrit par un expert. Sont par ailleurs données des informations sur les programmes de recherche en cours, les laboratoires de diagnostic, les associations de malades et les consultations spécialisées, ainsi que des informations sur les médicaments orphelins qui servent à leur traitement et des adresses d'autres sites Internet consacrés à ces maladies dans le monde.

\section{Qui utilise Orphanet? (données concernant la France seulement)}

Le site actuel reçoit une moyenne de 2000 visiteurs uniques par jour qui font une dizaine de requêtes par visite. Le nombre de pages lues chaque mois est de 600000 . Les visites proviennent de 90 pays dont $50 \%$ de France. Ceci s'explique par le fait que les informations sur les services concernaient exclusivement la France, depuis peu les autres pays européens ont commencé à insérer leurs données.

L'analyse des visiteurs du site actuel montre que 10\% sont des personnes intéressées aux maladies rares (sans autre lien particulier avec ces maladies), 13\% sont des personnes atteintes d'une maladie rare, $28 \%$ des personnes de la famille ou de l'entourage proche d'une personne atteinte, $28 \%$ des médecins, $5 \%$ des chercheurs, $16 \%$ d'autres professionnels de la santé, de l'industrie ou des médias (statistiques françaises).

\section{Comment est géré Orphanet?}

En France, Orphanet est géré par trois comités:

Le Comité d'Orientation est chargé de suivre le développement du projet Orphanet et de donner son avis sur l'adaptation de la base de données aux besoins des différents utilisateurs, la nature des informations proposées par cette base, le respect des règles de secret médical et des règles déontologiques en matière d'information médicale, les conditions d'utilisation notamment sur les modalités d'accès et les aspects financiers du projet. Le Comité d'Orientation est présidé en alternance par le Directeur Général de la Santé et le Directeur Général de l'INSERM.

Le Comité Scientifique Suisse est chargé d'expertiser et de valider les informations figurant dans la base de données. Il est composé d'experts désignés en raison de leur compétence scientifique par le Comité d'Orientation.

En Suisse: comité scientifique constitué par un médecin représentant des présidents des différentes sociétés suisses qui s'occupe de la vérification des donnés suisses à rentrer dans la base de données. Un webmaster pour la page nationale a un traducteur pour les langues nationales suisses.

Le Comité Editorial est chargé de nommer les experts qui rédigent les textes sur chaque maladie et de valider ces textes avant leur mise en ligne.

\section{Qu'est-ce qu'une maladie rare?}

\section{Quelle est la définition d'une maladie rare?}

Les maladies dites rares sont celles qui touchent un nombre restreint de personnes en regard de la population générale. Le seuil admis en Europe est d'une personne atteinte sur 2000, soit pour la France moins de 30000 personnes pour une maladie donnée. Le statut de maladie rare peut être instable. Pendant plusieurs dizaines d'années le SIDA a été une maladie rarissime, puis rare, maintenant fréquente dans certaines populations. Une maladie génétique ou une maladie virale peut être rare dans une région et fréquente dans une autre. La lèpre est une maladie rare en France, mais fréquente en Afrique centrale. La thalassémie, une anémie d'origine génétique, est rare dans le Nord de l'Europe, alors qu'elle est fréquente autour de la Méditerranée. La «maladie périodique» est rare en France, mais courante en Arménie. Il y a également des maladies fréquentes qui ont des variantes rares. 


\section{Combien $y$-a-t-il de maladies rares?}

Les maladies rares se comptent certainement par milliers. A l'heure actuelle, on a déjà dénombré 6000-7000 maladies génétiques et 5 nouvelles sont décrites chaque semaine dans la littérature médicale. Le nombre des maladies rares dépend aussi de la précision de la définition de ce qu'est une maladie. Jusqu'à présent, en médecine, une maladie était définie comme une altération de l'état de santé, se présentant en une configuration unique de symptômes dont le traitement est unique. Que la configuration soit considérée comme unique dépend de notre finesse d'analyse. Plus celle-ci est fine, plus nous percevons de nuances. Il se peut que toutes les maladies fréquentes se décomposent, dans quelques années, en plusieurs dizaines ou centaines de maladies rares.

\section{Quelle est l'origine des maladies rares?}

Si presque toutes les maladies génétiques sont des maladies rares, toutes les maladies rares ne sont pas génétiques. Il $\mathrm{y}$ a des maladies infectieuses très rares par exemple, ainsi que des maladies auto-immunes. Pour un grand nombre de maladies, la cause demeure inconnue à ce jour.

\section{Quelles sont les caractéristiques des maladies rares?}

Les maladies rares sont des maladies graves, chroniques, évolutives où le pronostic vital est souvent en jeu. L'atteinte peut être visible dès la naissance ou l'enfance, comme par exemple pour l'amyotrophie spinale infantile, la neurofibromatose, l'ostéogenèse imparfaite, les chondrodysplasies ou le syndrome de Rett. Cependant plus de 50\% des maladies rares apparaissent à l'âge adulte, comme la maladie de Huntington, la maladie de Crohn, la maladie de Charcot-Marie-Tooth, la sclérose latérale amyotrophique, le sarcome de Kaposi ou le cancer de la thyroïde. Les maladies rares souffrent d'un déficit de connaissances médicales et scientifiques. Longtemps ignorées des médecins, des chercheurs et des politiques, il n'existait pas de politique de recherche les concernant, jusqu'à un passé très récent. Pour la plupart d'entre elles il n'existe pas de traitement curatif, mais des soins appropriés peuvent améliorer la qualité de vie et prolonger la durée de vie. Des progrès spectaculaires ont déjà été accomplis pour certaines maladies, montrant bien qu'il ne faut pas baisser les bras, mais au contraire poursuivre et intensifier l'effort de recherche et de solidarité sociale.

Quelles sont les conséquences médico-sociales de la rareté de ces maladies?

Les personnes atteintes par ces maladies rencontrent toutes des difficultés similaires dans leur parcours vers un diagnostic, pour obtenir de l'information et pour être orientées vers les professionnels compétents. L'accès à des soins de qualité, la prise en charge globale sociale et médicale de la maladie, la coordination des soins hospitaliers et de ville, l'autonomie et l'insertion sociale, professionnelle et citoyenne posent également problème. Beaucoup de maladies rares s'accompagnent de déficits sensoriel, moteur, mental, et parfois de stigmates physiques. Les personnes atteintes de maladies rares sont plus vulnérables, sur le plan psychologique, social, économique et culturel. Ces difficultés pourraient être réduites par une politique adaptée. Faute de connaissances scientifiques et médicales suffisantes, un grand nombre de malades n'est pas diagnostiqué. Leur maladie demeure inconnue. Au mieux, ces personnes sont alors prises en charge sur la base de l'expression de leurs symptômes. Ce sont ces personnes qui souffrent le plus de difficultés de prise en charge.

Tous les malades et leur familles décrivent un parcours du combattant pour être écoutés, pour s'informer et pour être orientés vers les équipes compétentes, lorsqu'elles existent, afin de poser le bon diagnostic. Il en résulte un gaspillage en délais inutiles, en multiplication des consultations et en consommation de soins et de médicaments inappropriés, voire nuisibles à la santé. Rarement l'annonce du diagnostic est bien faite, en dépit de progrès certains réalisés depuis 10 ans. Tous les malades et les familles décrivent une annonce difficile, peu informative et peu construite. Cette difficulté est partagée par les soignants, qui ne sont ni organisés, ni formés aux bonnes pratiques d'annonce du diagnostic. Les malades et leur famille décrivent, même après le diagnostic, des épisodes de soins gravement inappropriés. L'état de l'art dans la prise en charge n'est pas diffusé. Il existe peu de référentiels de bonnes pratiques cliniques. Le travail et le savoir des médecins sont isolés quand ils devraient être en réseau et partagés. La segmentation en spécialités médicales ne permet pas une prise en charge globale des malades atteints de maladies qui touchent plusieurs organes. Ces maladies sont trop rares pour qu'existe dans chaque région une expertise satisfaisante. Les différences d'accès aux soins sont liées d'une part à la plus ou moins grande capacité des médecins à travailler en réseau et d'autre part à une sectorisation administrative, source d'inégalités supplémentaires. Famille et soignants se plaignent unanimement de la lourdeur, de la multiplication et de la fréquence des démarches administratives. Des inégalités certaines existent dans l'allocation des aides financières, des revenus de substitution et des remboursements de soins, avec de fortes disparités régionales. Du fait de la rareté des maladies et des lieux de soins, les frais engagés sont souvent supérieurs à ceux des autres maladies. Une partie significative de ces frais est supportée financièrement par les familles.

\section{Peut-on espérer des progrès dans le diagnostic et le}

traitement de ces maladies?

Pour toutes ces maladies, de grands espoirs et de profonds changements sont attendus des progrès scientifiques, en particulier des résultats du programme «Génome Humain». Plusieurs centaines de ces maladies peuvent être maintenant diagnostiquées par un test biologique. Les connaissances sur l'histoire naturelle des maladies progressent avec la création d'observatoire pour certaines d'entre elles. Les chercheurs travaillent de plus en plus souvent en réseau pour partager les résultats de leurs recherches et avancer plus efficacement. De nouveaux espoirs naissent avec les perspectives offertes par une politique sur les médicaments orphelins.

Le soutien à cet effort de recherche est variable d'un pays à l'autre, peu de pays ayant encore une politique de soutien ciblé à la recherche sur les maladies rares. 


\section{Tribune libre}

\section{La politique européenne dans le domaine des maladies rares}

Deux programmes de la Commission européenne sont directement liés aux maladies rares:

Programme européen «Maladies rares» (1999-2003), http://europa.eu.int/comm/health/ph/programmes/rare/index_en.htm;

Programme européen pour la recherche et le développement technologique (1998-2002), http://www.cordis.lu/life/home. html.

\section{Les associations de patients}

En France, l'Alliance Maladies Rares, créée le 24 février 2000 , est le collectif national qui réunit les associations et les personnes concernées par les maladies rares.

EURORDIS (European Organisation for Rare Disorders) est l'association européenne des maladies rares. EAGS (European Alliance of Parents and Patients Organisations for Genetic Services and Innovation in Medicine).

\section{Consultations génétiques et laboratoires en Suisse}

Consultations génétiques, analyses caryotypiques, analyses de l'ADN et diagnostic prénatal

\section{Basel}

Abteilung für Medizinische Genetik, Universitätskinderspital beider Basel

Römergrasse 8, CH-4005 Basel

Tel. 06168564 32, Fax 0616856011

Forschungsgruppe Humangenetik, Departement Forschung CH-4031 Basel

Tel. 06126523 62, Fax 0612652350

Bern

Medizinische Genetik, Universitätskinderklinik

Inselspital

CH-3010 Bern

Tel. 03163294 82, Fax 0316329748

Genève

Division de Génétique Médicale

CMU, Rue Michel-Servet 1, CH-1211 Genève 4

Tél. 02237957 07/31, Fax 0223795706

\section{Lausanne}

Service de Génétique Médicale, CHUV

CH-1011 Lausanne

Tél. 02131433 78, Fax 0213143385

\section{Zürich}

Institut für Medizinische Genetik der Universität Zürich

Rämistrasse 74, CH-8001 Zürich

Tel. 0163425 21, Fax 0216344916

\section{Adresses d'autres laboratoires}

Basel

Institut Dr. Vollier AG

Spalenring 145/147, CH-4002 Basel

Tel. 06148611 11, Fax 0614820030
Universitätsfrauenklinik, Hormonlabor

Postfach, CH-4031 Basel

Tel. 0612652525

Bern

Pathologisches Institut der Universität Bern,

Zytogenetisches Labor

Freiburgstrasse 30, CH-3010 Bern

Tel. 0316323207

Inselspital, Chemisches Zentrallabor

CH-3010 Bern

Tel. 0316322201

Düdingen

Medizinische Laboratorien MCL

Postfach, CH-3186 Düdingen

Tel. 0264927245,0264927239

Genève

Policlinique Universitaire de Gynécologie et d'Obstétrique,

Laboratoire d'Hormonologie de la Maternité

Boulevard de la Cluse 32, CH-1205 Genève

Tél. 02238244 00, Fax 0223724400

Division d'Hématologie, Hôpital Cantonal

Universitaire de Genève

Rue Micheli-du-Crest 24, CH-1211 Genève 4

Tél. 02237239 47, Fax 0223727288

Diagnostic prénatal de thalassémie et des hémoglobines anormales, Laboratoire de biologie moléculaire

Tél. 0223723932

Unité d'identification des prédispositions génétiques au cancer Division d'Oncologie, HUG

Rue Micheli-du-Crest 24, CH-1211 Genève 4

Tél. 02237298 53, Fax 0227729858

Lausanne

Laboratoire de Service de Gynécologie et d'Obstétrique, CHUV CH-1011 Lausanne

Tél. 0213142595

Laboratoire AMS

Rue du Liseron 5, CH-1000 Lausanne 6

Tél. 0216174144

Sion

Laboratoire d'ADN, Institut Central des Hôpitaux Valaisans

Avenue Grand-Champsec 86, CH-1951 Sion

Tél. 0273245351

St. Gallen

Enzym-Labor Dr. H. Weber AG

Müller-Friedberg-Strasse 6, CH-9000 St. Gallen

Tel. 07122744 44, Fax 0712274445

Zürich

Genetica AG, Zytogenetisches Labor

Weinbergstrasse 9, CH-8001 Zürich

Tel. 012519094

Medizinische Universitätsklinik, Abteilung für

Klinische Immunologie

Häldeliweg 4, CH-8044 Zürich

Tel. 012553586

Universitätskinderklinik, Onkologielabor

Steinwiesstrasse $75, \mathrm{CH}-8032$ Zürich

Tel. 0126671 11, Fax 012667171 